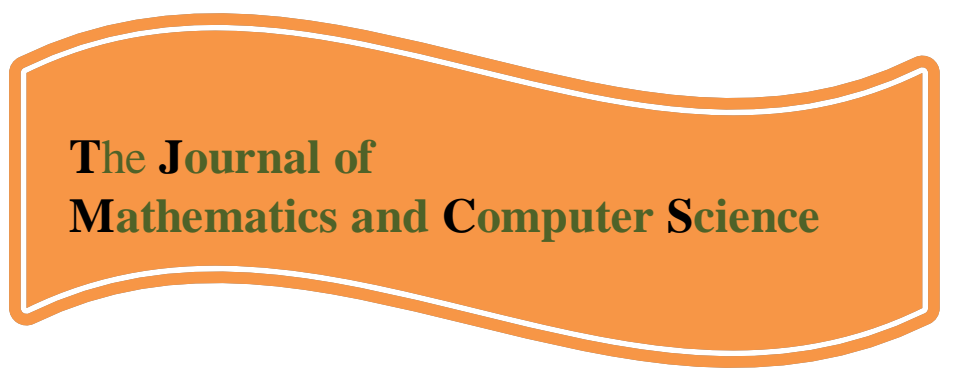

Available online at

http://www.TJMCS.com

The Journal of Mathematics and Computer Science Vol .1 No.4 (2010) 273-286

\title{
Chaos Synchronization of two Uncertain Chaotic System Using Genetic Based Fuzzy Adaptive PID Controller
}

\author{
Yaghoub Heidari1, ${ }^{*}$, Soheil Salehi Alashti², Rouhollah Maghsoudi3, \\ Department of Electrical, Nour Branch, Islamic Azad University, Nour, Iran \\ Babol Noshirvany University of Technology, soheilsalehy@stu.nit.ac.ir \\ Department of Computer, Nour Branch, Islamic Azad University, Nour, Iran
}

Received: August 2010, Revised: October 2010

Online Publication: December 2010

\begin{abstract}
A Genetic based Fuzzy PID controller has been proposed to synchronization task of chaotic systems in which one system has been considered as "master" whilst the other system has been treated as "slave" (a perturbed system with uncertainty and disturbance). Three PID control gains $k_{p}, k_{i}$, and $k_{d}$, will be updated online. An adequate adaptation mechanism is used to minimize the sliding surface error with appropriate adaptive law. Using the gradient method, coefficients of the PID controller are updated. A supervisory controller has also been used to provide the stability. The proposed method has been found with a significant performance when it was implemented on the Van Der Pol oscillator chaotic equations.
\end{abstract}

Keywords: Fuzzy, Chaos synchronization; supervisory controller; Van der Pol; Adaptive Control.

\footnotetext{
${ }^{1, *}$ Corresponding Author.

E-mail address: masudheidari@yahoo.com (Phone: +98 122625 4590)

2- Babol Noshirvany University of Technology,

3- Computer Department of Islamic Azad University of Nour (Phone: +98 122625 4590)
} 


\section{Introduction}

Feasibility of control and synchronizing chaotic systems in various types has attracted significant interest in the last few years [1-6]. Chaos is a peculiar random-like behavior in deterministic systems. In order to achieve the synchronization, a closed loop nonlinear control system, which obtains signals from the master and controls the slave accordingly, will be designed. In the literature, different non linear methods such as periodic parametric perturbation $[7,8]$, driveresponse synchronization [9], adaptive control [10-14], variable structure (or sliding mode) control [15-17], backstepping control [18, 19], and $\mathrm{H}_{\infty}$ control [20], have been used for synchronization. Chaos synchronization can also be considered as a design problem of a feedback law for an observer, using known information of the plant. This is to ensure that the controlled receiver is synchronized with the transmitter.

In [21] an adaptive robust PID controller of a chaotic system is presented. The aim is to improve and develop such a method to synchronize an unknown slave dynamic with a master system. A fuzzy sliding-mode control (FSMC) strategy has been proposed for uncertain chaotic systems in [22], in which a fuzzy controller is used to replace the discontinuous sign function of the reaching law in traditional sliding-mode control (SMC). An active sliding mode control for synchronizing two chaotic systems with parametric uncertainty was proposed in [23]. An algorithm to determine parameters of active sliding mode controller, during synchronization of different chaotic systems has been studied in [24]. In [25] an adaptive sliding mode controller is presented for class of master-slave chaotic synchronization systems with uncertainties. Fuzzy sliding mode control has been used in many papers [26-29].

In this paper, parameters of PID controller are tuned adaptively by the gradient method, according to a proper designed sliding surface. To guarantee the convergence, a supervisory control using a suitable Lyapunov function has been designed. As a side effect, the signum function in the supervisory controller can cause chattering phenomenon in the control signal. To reduce this effect a Genetic Based Fuzzy Controller (GBFC) will be proposed. The synchronization procedure and the stability requirements will be shortly introduced.

This paper is organized as follows: In section 2, a quick introduction to the proposed method for synchronization of the chaotic system in presence of uncertainty is addressed. Section 3 is devoted on investigation of the application of the proposed method on chaotic gyroscope, described by Van Der Pol oscillator dynamic via simulation, when the system is perturbed with uncertainties. Finally, the result analysis and conclusion will be addressed in section 4 .

\section{Synchronization of uncertain chaotic system using adaptive PID controller}

Model reference adaptive control or model following task is one of usual tasks in control engineering which is called Master-Slave problem in electrical engineering. The ultimate goal of this task is to design a mechanism, which forces the slave, to follow the master regardless of some possible incompatibility. If the slave is chaotic, uncertain or of a reduced order, the synchronization problem takes a complex form. Let us consider the following dynamic as a master:

$\dot{x}_{1}=x_{2}$

$\dot{x}_{2}=f(X, t)$

Where, $X$ is a two dimensional state vector as $X=\left[x_{1}, x_{2}\right]^{T}$ and $f($.$) is an unknown function.$ Suppose the following dynamic as slave in the synchronization task: 
$\dot{y}_{1}=y_{2}$

$\dot{y}_{2}=f(Y, t)+\Delta f(Y, t)+d(t)+u(t)$

$y=\left[y_{1}, y_{2}\right]^{T}$ is the output vector, $\Delta f($.$) and d(t)$ denote uncertainty and disturbance respectively.

The control signal $u(t)$ has a duty to synchronize the slave system (2) with the master dynamic in (1). A schematic diagram of the synchronization, which is eventually inspired from the adaptive control terminology, is shown in Figure (1). The difference between master and slave will be minimized via an adaptive controller. The synchronizer designation consists of the following sections:

1. PID controller

2. A supervisory controller to guarantee the stability.

3. A method to update PID coefficients. The gradient or a sliding surface will be such an update mechanism.

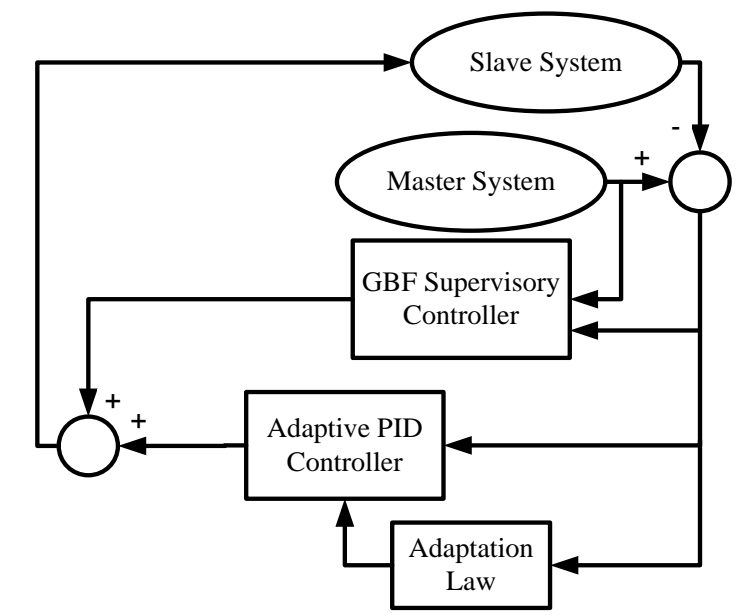

Figure 1: Schematic diagram of a synchronization mechanism

The error vector of two states is defined for by $e_{i}=x_{i}-y_{i}, i=1,2$. Suppose that we can choose a gain vector $\mathrm{K}=\left[\mathrm{k}_{0}, \mathrm{k}_{1}\right]^{\mathrm{T}}$ such that roots of $s^{2}+k_{2} s+k_{1}=0$ are in the left hand side of the s-plane. A feedback linearization technique is a possible choice, to combat both uncertainty and disturbance, which is as follows:

$\ddot{e}_{1}+k_{2} \dot{e}_{1}+k_{1} e_{1}=0 \rightarrow \dot{x}_{2}-\dot{y}_{2}+k_{2} e_{2}+k_{1} e_{1}=0$

$u_{F . L .}(t)=-f(Y, t)-\Delta f(Y, t)-d(t)+\dot{x}_{2}+k_{2} e_{2}+k_{1} e_{1}$

It is of the goal to reduce the error asymptotically. Therefore, the slave must follow the master dynamically. It should be noted that $2^{\text {nd }}$ system is unknown and the states and error are the only available signals. These may have been infected by some uncertainties and perturbations. The following assumptions are required to design an adaptive PID.

Assumption 1. Let the constraint set $\Omega_{y}$ for the state $X$ is defined as:

$\Omega_{y}=\left\{Y \in \mathbb{R}^{2}:\|Y\| \leq M_{y}\right\}$, 
Where, $M_{y}$ is a pre-specified upper limit. It is desired that the state trajectory of system $X$ never reaches to the boundary, during the control action. For simplicity in analysis, we choose $M_{y} \geq\|X\|_{\infty}$. Let us assume the control input of the form:

$u=u_{P I D}+u_{S}$

where, $u_{P I D}$ denotes the signal produced by PID controller which is defined as follows:

$u_{P I D}=K_{P} e(t)+K_{I} \int_{0}^{t} e(\tau) d \tau+K_{D} \frac{\partial e(t)}{\partial t}$

Furthermore, $u_{S}$ supervisory controller signal [21], is added to guarantee the stability. Then a sliding mode control is proposed. Accordingly, a proper adaptation law, based on the gradient method, is designed to minimize the error. Let us redefine the state in equation (3) using the control law in equation (5), by the following statement:

$$
\begin{aligned}
\dot{y}_{1} & =y_{2} \\
\dot{y}_{2} & =f(Y, t)+\Delta f(Y, t)+d(t)+u_{P I D}+u_{s} \\
& =f(Y, t)+\Delta f(Y, t)+d(t)+u_{P I D}+u_{s}+u_{F . L .}-u_{F . L .} . \\
& =\dot{x}_{2}+k_{2} e_{2}+k_{1} e_{1}+\left[u_{P I D}+u_{s}-u_{F . L .}\right]
\end{aligned}
$$

This immediately follows that:

$\dot{e}_{1}=e_{2}$

$\dot{e}_{2}=-k_{2} e_{2}-k_{1} e_{1}-\left[u_{P I D}+u_{s}-u_{F . L .}\right]$

The following gain vectors:

$A=\left[\begin{array}{rr}0 & 1 \\ -k_{1} & -k_{2}\end{array}\right], B=\left[\begin{array}{l}0 \\ 1\end{array}\right]$

Establish the state feedback. Therefore, the error dynamic will be of the form:

$\dot{E}=A E+B\left[u_{F . L .}-u_{P I D}-u_{S}\right]$

Where, $E$ is $\left[e_{1}, e_{2}\right]$. The energy of the system will be candidate as a Lyapunov function according to:

$V=\frac{1}{2} E^{T} P E$

Where $p$ is a symmetric and positive definite matrix. This will be found via following Lyapunov equation:

$$
A^{T} P+P A=-Q
$$

Furthermore, $Q$ is also a symmetric and positive definite matrix. This may be designed by the user in advance, to meet such other criteria. Definition of $V_{M}$ as:

$V_{M}=\frac{1}{2} \min (\lambda(P))\left(M_{y}-\|X\|_{\infty}\right)^{2}$

leads us to the following equation:

$$
\begin{aligned}
V & \geq \frac{1}{2} \min (\lambda(P))\|E\|^{2} \geq \frac{1}{2} \min (\lambda(P))(\|Y\|-\|X\|)^{2} \\
& \geq \frac{1}{2} \min (\lambda(P))\left(M_{y}-\|X\|_{\infty}\right)^{2}=V_{M}
\end{aligned}
$$


Where $\min (\lambda(P))$ denotes the minimum value of eigenvalue of matrix $P$. For the case $V<V_{M}$ one obtains $\|X\|<M_{y}$. By taking the time derivative of (11) and using (10) leads us to:

$$
\begin{aligned}
\dot{V}_{e} & =\frac{1}{2} E^{T}\left(A^{T} P+P A\right) E+E^{T} P B\left[u_{F . L .}-u_{P I D}-u_{S}\right] \\
& =-\frac{1}{2} E^{T} Q E+E^{T} P B\left[u_{F . L .}-u_{P I D}-u_{S}\right] \\
& \leq-\frac{1}{2} E^{T} Q E+\left|E^{T} P B\right|\left(\left|u_{F . L .}\right|+\left|u_{P I D}\right|\right)-E^{T} P B u_{S}
\end{aligned}
$$

An achievable approximation of inequalities $|f()|<.f_{u}(),.|\Delta f()|<.\Delta f_{u}($.$) and |d(t)|<\delta$ with aiding equation (3) provides a lower limit for the feedback linearization law. This immediately follows that:

$$
u_{F . L .}(t) \leq f_{u}+\Delta f_{u}+\delta+\left|\dot{x}_{2}\right|+\left|k_{2} e_{2}+k_{1} e_{1}\right|
$$

For the other case when $V>V_{M}$, the following supervised controller guarantees the necessity condition of $\dot{V}<0$.

$$
u_{S}= \begin{cases}0, & , V \leq V_{M} \\ \operatorname{sgn}\left(E^{T} P B\right)\left\lfloor f_{u}+\Delta f_{u}+\delta+\left|\dot{x}_{2}\right|+\left|k_{2} e_{2}+k_{1} e_{1}\right|\right\rfloor, & V>V_{M}\end{cases}
$$

In order to have an adaptation mechanism to update PID coefficients, reference signal $y_{r}$ can be defined as:

$$
\dot{y}_{r}=\dot{x}_{2}+k_{2} \dot{e}_{1}+k_{1} e_{1}
$$

and the sliding surface by:

$$
S=y_{2}-y_{r}
$$

When the sliding mode is activated i.e. $S=0$, therefore we have:

$$
y_{2}=y_{r}
$$

Replacing equation (20) in (18) approaches:

$$
\ddot{e}_{1}+k_{2} \dot{e}_{1}+k_{1} e_{1}=0
$$

A necessary and sufficient condition for the convergence needs the error to approach zero when the time tends to infinity. Furthermore, to meet the sliding condition, the following Lyapunov function will be candied to realize a stable controller.

$$
V=\frac{1}{2} S^{2}
$$

Therefore, differentiation of $V$ would be as:

$$
\dot{V}=S \dot{S}<0
$$

It guarantees the convergence when $S(t) \rightarrow \infty$ as $t \rightarrow \infty$. A proper adaptation mechanism needs to minimize the sliding condition $S \dot{S}$. The gradient search algorithm is calculated in the opposite direction of the energy flow. Therefore, the convergence of the system can be obtained by a satisfactory adjustment of PID coefficients. Furthermore, it is quite intuitive to choose $S \dot{S}$ as an error function. From (19) and using (2), obtains:

$$
\dot{S}=\dot{y}_{2}-\dot{y}_{r}=f(Y, t)+\Delta f(Y, t)+d(t)+u(t)-\dot{y}_{r} \text {. }
$$

Replacing (5) in (24) and multiplying both sides of (24) in $S$, there will be:

$$
S \dot{S}=S\left[f(Y, t)+\Delta f(Y, t)+d(t)+u(t)-\dot{y}_{r}\right] .
$$


Applying the gradient method and the chain principle to equations (6) and (25), the adaptation law for determining PID parameters will be obtained as:

$$
\begin{aligned}
& \dot{K}_{P}=-\gamma \frac{\partial S \dot{S}}{\partial K_{P}}=-\gamma \frac{\partial S \dot{S}}{\partial u_{P I D}} \frac{\partial u_{P I D}}{\partial K_{P}}=-\gamma S e_{1}(t) \\
& \dot{K}_{I}=-\gamma \frac{\partial S \dot{S}}{\partial K_{I}}=-\gamma \frac{\partial S \dot{S}}{\partial u_{P I D}} \frac{\partial u_{P I D}}{\partial K_{I}}=-\gamma S \int_{0}^{t} e_{1}(\tau) d \tau \\
& \dot{K}_{D}=-\gamma \frac{\partial S \dot{S}}{\partial K_{D}}=-\gamma \frac{\partial S \dot{S}}{\partial u_{P I D}} \frac{\partial u_{P I D}}{\partial K_{D}}=-\gamma S \frac{\mathrm{d}}{\mathrm{d} t} e_{1}(t)
\end{aligned}
$$

where, $\gamma>0$ is the learning rate. It should be noted that selection of $\gamma$ and/or the initial setting of PID coefficients is very crucial for the stability [21]. The discontinuity of the sign function in (17) causes the chattering phenomenon. Using saturation instead of a simple sign function in (31), to reduce the chattering, in (17) obtains:

$$
\begin{aligned}
& u_{S}=K \operatorname{sat}(\psi)\left[f_{u}+\Delta f_{u}+\delta+\left|\dot{x}_{2}\right|+\left|k_{2} e_{2}+k_{1} e_{1}\right|\right] \\
& \psi=E^{T} P B
\end{aligned}
$$

and

$$
\operatorname{sat}(\psi / \Phi)= \begin{cases}\operatorname{sgn}(\psi / \Phi) & \text { if }|\psi / \Phi| \geq 1 \\ \psi / \Phi & \text { if }|\psi / \Phi|<1\end{cases}
$$

However to have a better compromise between small chattering and good tracking precision in the presence of parameter uncertainties, a fuzzy controller is proposed.

\section{Fuzzy control}

The main advantage of the fuzzy controller is its heuristic design procedure as a model-free approach. The combination of fuzzy control strategy and (29) becomes a feasible approach to preserve advantages of these two approaches $[30,31]$ especially to reduce the chattering. A fuzzy controller is introduced to develop (29) control law. The IF-THEN rules of fuzzy controller can be described as:

$$
\begin{aligned}
& \text { R1: If } \psi \text { is NB then K is PB } \\
& \text { R2: If } \psi \text { is NM then K is PM } \\
& \text { R3: If } \psi \text { is NS then K is PS } \\
& \text { R4: If } \psi \text { is ZE then K is ZE } \\
& \text { R5: If } \psi \text { is PS then K is NS } \\
& \text { R6: If } \psi \text { is PM then K is NM } \\
& \text { R7: If } \psi \text { is PB then K is NB }
\end{aligned}
$$

Where NB, NM, NS, ZE, PS, PM, PB are the linguistic terms of antecedent fuzzy set. They mean Negative Big, Negative Medium, Negative Small, Zero, Positive Medium Positive Small and Positive Big, respectively. The fuzzy membership function for each fuzzy term should be a proper design factor in the fuzzy control problem. A general form is used to describe these fuzzy rules as:

$\mathrm{Ri}$ : If $\psi$ is $\mathrm{Ai}$, then $\mathrm{K}$ is $\mathrm{Bi}$ 
Where Ai has a triangle membership function (depicted in figure 2) and Bi is a fuzzy singleton. The modified controller (29), invites an idea to restrict the width of boundary layer $\Phi$, and uses a continuous function (31) to smoothen the control action. Therefore, the problem of the discontinuousness of the sign function can be solved, and the chattering phenomena will be decreased. From the control point of view, the parameters of structures should be automatically modified by evaluating the results of fuzzy control in (32). Hitting time and chattering phenomenon are two important factors that influence the performance of proposed Controller. The width of boundary layer, $\Phi$, will influence the chattering magnitude of control signal, and the gain $\mathrm{K}$, will influence how soon the states of Slave reach to that of in Master.

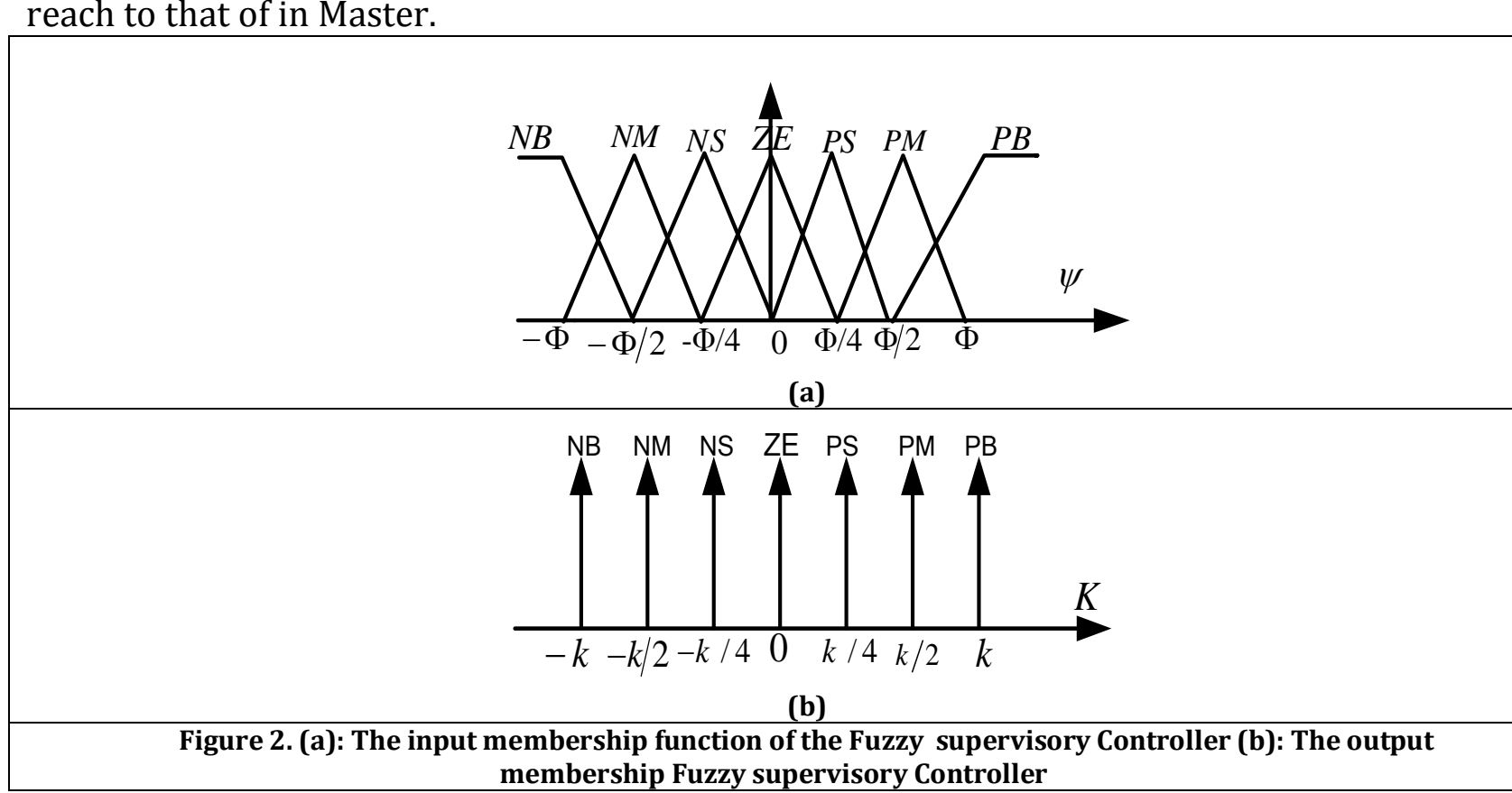

The reaching time can also be reduced via a suitable selection of parameter $K$. GA is used to search for a best fit for $k, \phi, k_{1}, k_{2}$ parameters in (29). The tracking error and existence of chattering in the controlled response are chosen as a performance index to select the parameters. The proposed fitness function is defined in such a way that the selected parameters can force the state to reach together fast and then keep the control signal with less chattering and synchronization error. In this manner, GA is used to search the parameter space to find appropriate values of the parameters, $k, \phi, k_{1}, k_{2}$ in (29). A fitness function is defined as follows:

$$
y=\int\left[W_{1}\left(e^{2}{ }_{1}+e^{2}{ }_{2}\right)+W_{2}(\psi)^{2}\right] d t
$$

where $e_{1}, e_{2}$ is defined in (3), and $\mathrm{W} 1$ and $\mathrm{W} 2$ are the weight factors. The design parameters of the GBFCassociated with the above control rules are specified as follows:

Population size $=60 \quad$ Crossover probability $=0.8$

Generations $=70 \quad$ Mutation probability $=0.02$

$k$ belongs to $[0,20] \quad \phi$ belongs to $[0,10]$ 
These are popular setting and can be easily found by a minor modification. The Fuzzy designed controller has applied to some case studies in the next sections.

\section{Simulation}

\subsection{Synchronization of Uncertain Hard Spring $\Phi 6$-Van der Pol Oscillator}

\subsubsection{System description}

Consider the following system namely $\Phi^{6}$ - Van Der Pol oscillator [34] as another case study:

$$
\ddot{x}-\mu\left(1-x^{2}\right) \dot{x}+\omega_{0}^{2} x+\alpha x^{3}+\lambda x^{5}=f_{0} \cos \omega t
$$

The above system for $f_{0}=0$, is a complete oscillatory system. Increasing $f_{0}$ appears chaotic characteristic of the system [34]. In Figure (3), phase portrait of the chaotic system namely $\varphi^{6}-$ Van Der Pol oscillator with $f_{0}=4.5$ has been shown. Simultaneously, parameters of system have been selected as $M=0.4, \alpha=1, \lambda=0.1, w_{o}=0.46, w=0.86$. In Figure (3), chaotic behavior of the system is completely evident. Correspondingly, parameter $f_{0}$ is treated and designed as an uncertainty of the system. To show the effectiveness of the proposed controller, the procedure is implemented here.

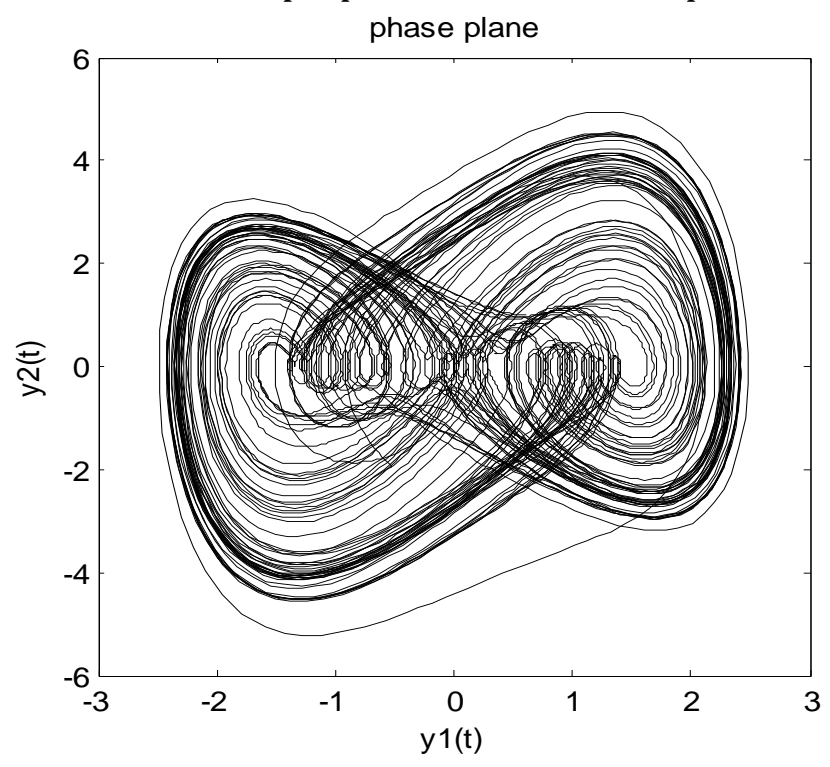

Figure 3: Phase portrait of $\Phi 6$-Van Der Pol oscillator

\subsubsection{Implementation}

The master system is defined by the following dynamic:

$$
\left\{\begin{array}{l}
\dot{x}_{1}=x_{2} \\
\dot{x}_{2}=\mu\left(1-x_{1}^{2}\right) x_{2}-\omega_{0}^{2} x_{1}-\alpha x_{1}^{3}-\lambda x_{1}^{5}
\end{array}\right.
$$

Similarly, the slave is defined as follows:

$$
\left\{\begin{array}{l}
\dot{y}_{1}=y_{2} \\
\dot{y}_{2}=\mu\left(1-y_{1}{ }^{2}\right) y_{2}-\omega_{0}^{2} y_{1}-\alpha y_{1}^{3}-\lambda y_{1}{ }^{5}+f_{0} \cos \omega t
\end{array}\right.
$$


Sets of initial conditions of master and slave systems are respectively defined as $x_{1}(0)=0.2, x_{2}(0)=-0.2$ and $y_{1}(0)=-0.5, y_{2}(0)=-2$. Considering the disturbance $d(t)=f_{0} \cos \omega t$ and $f\left(x_{1}, x_{2}\right)=M\left(1-y_{1}^{2}\right)-w_{0}^{2} y_{1}+\alpha y_{1}^{3}+\lambda y_{1}^{5}$, an upper limit of related functions are obtained as follows:

$$
\begin{aligned}
|f(.)| & =\left|\mu\left(1-y_{1}{ }^{2}\right) y_{2}-\omega_{0}^{2} y_{1}-\alpha y_{1}{ }^{3}-\lambda y_{1}{ }^{5}\right| \\
& \leq\left|\mu y_{2}\right|\left(1+y_{1}{ }^{2}\right)+\omega_{0}^{2}\left|y_{1}\right|+\left|\alpha y_{1}{ }^{3}\right|+\left|\lambda y_{1}{ }^{5}\right| \\
& |d(t)|=\left|f_{0} \cos \omega t\right| \leq\left|f_{0}\right|
\end{aligned}
$$

The value of $M_{y}$ is similarly selected to be large enough such that the system state x never reach the boundary of $\Omega_{y}$. Initial setting of PID coefficients are equal to $k_{p}(0)=1, k_{1}(0)=1, K_{D}(0)=1$ and the learning rate has been selected as $\gamma=1$. Also, $k_{1}$ and $k_{2}$ have been assigned as 0.5 and 2 respectively. In comparison with equation (9), the state feedback matrix will be found as:

$$
A=\left[\begin{array}{cc}
0 & 1 \\
-0.5 & -2
\end{array}\right]
$$

Regarding to equations (9) and (41), choosing $Q=\left[\begin{array}{ll}1 & 0 \\ 0 & 1\end{array}\right]$, yields the symmetric and definite positive matrix p as: $p=\left[\begin{array}{cc}2.375 & 1 \\ 1 & 0.75\end{array}\right]$. Parameters of the system, similar to section 3.2.1, have been selected as $f_{0}=4.5, M=0.4, \alpha=1, \lambda=0.1, \omega_{\circ}=0.46, w=0.86$. Applying the sign function (17) to the master and slave system of $\Phi 6$ - Van Der Pol results the oscillation, which have been shown in Figures (4) to (6). In Figure (4), synchronization of $x_{1}, y_{1}$ and $x_{2}, y_{2}$, in Figure (5), the sliding surface and ultimately in Figure (6). Simulation results show the chattering phenomenon in the control signal, this can wear and tear the actuators. To reduce this undesired effect in control signal the GBFC has applied to synchronize the master and slave system of $\Phi 6$ - Van Der Pol. The relevant responses are shown in Figure (7) to (10). Figure (7) shows the synchronization of $x_{1}, y_{1}$ and $x_{2}, y_{2}$, whereas, Figure (8) shows the sliding surface and ultimately in Figure (9), the control signal can be seen. The synchronization error has also been seen in Figure (10). From Figure (9) can be seen that the control signal is smoother than that of in the sign function application. It should be noted that in this case, the control i.e. $u(t)$, has been activated at $t=5 \mathrm{~s}$. The performance of the GBFC is seen much improved.

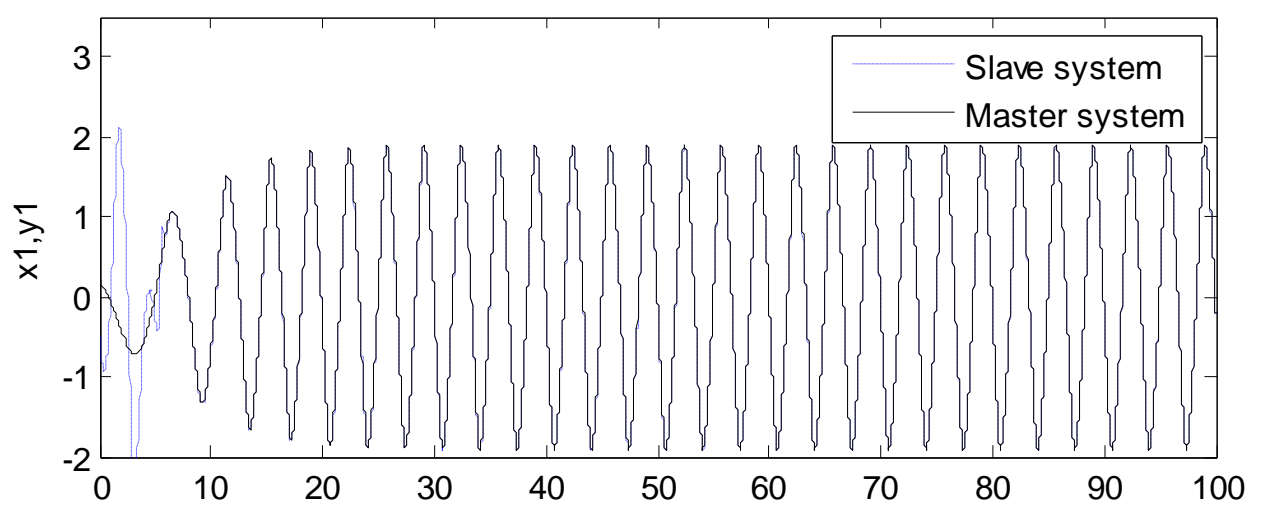




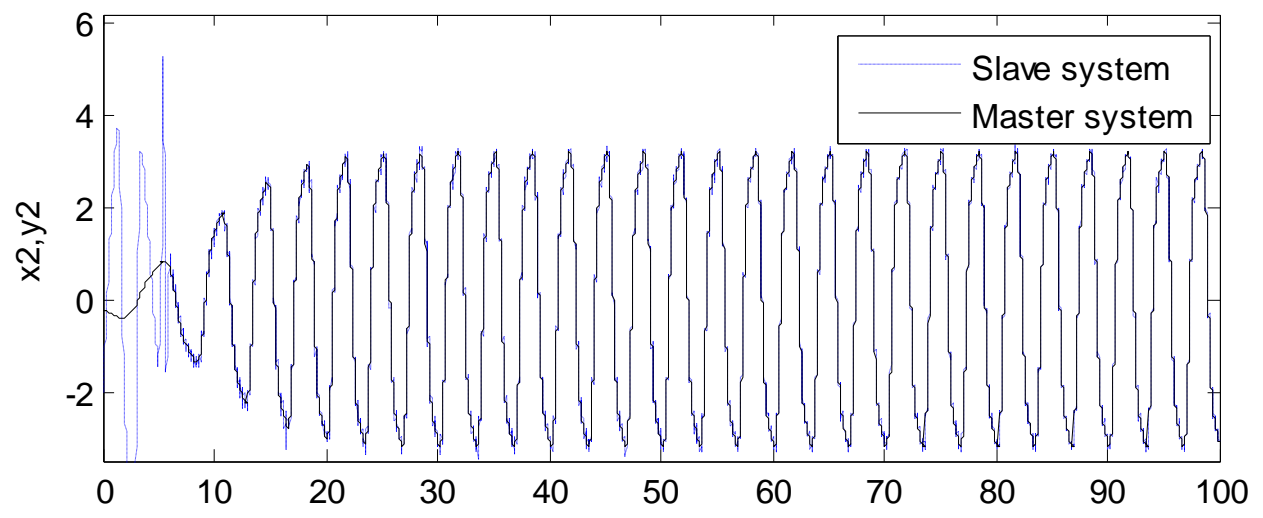

Figure 4: Time response of controlled chaotic $\Phi 6$-van der pol synchronization system
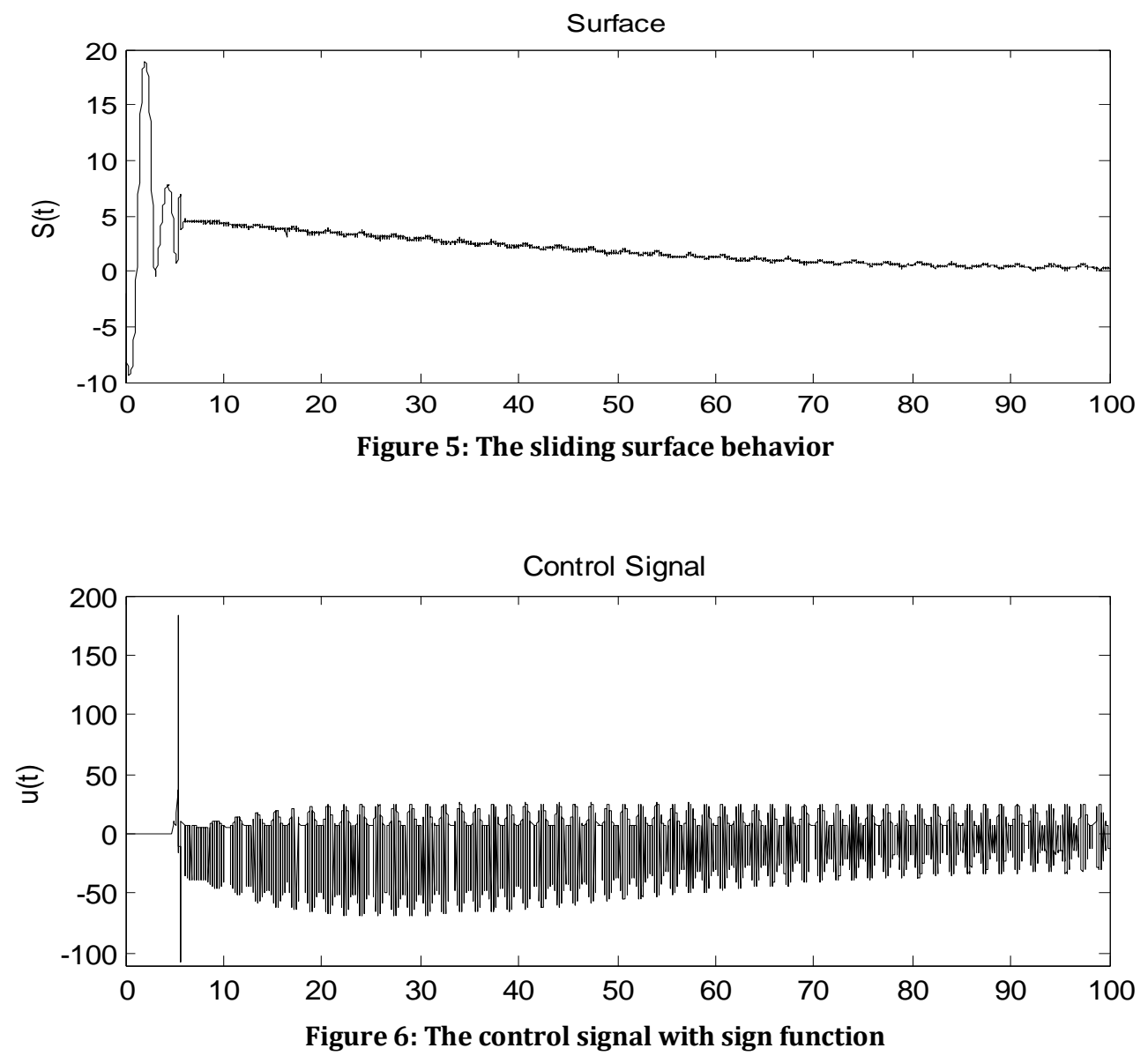

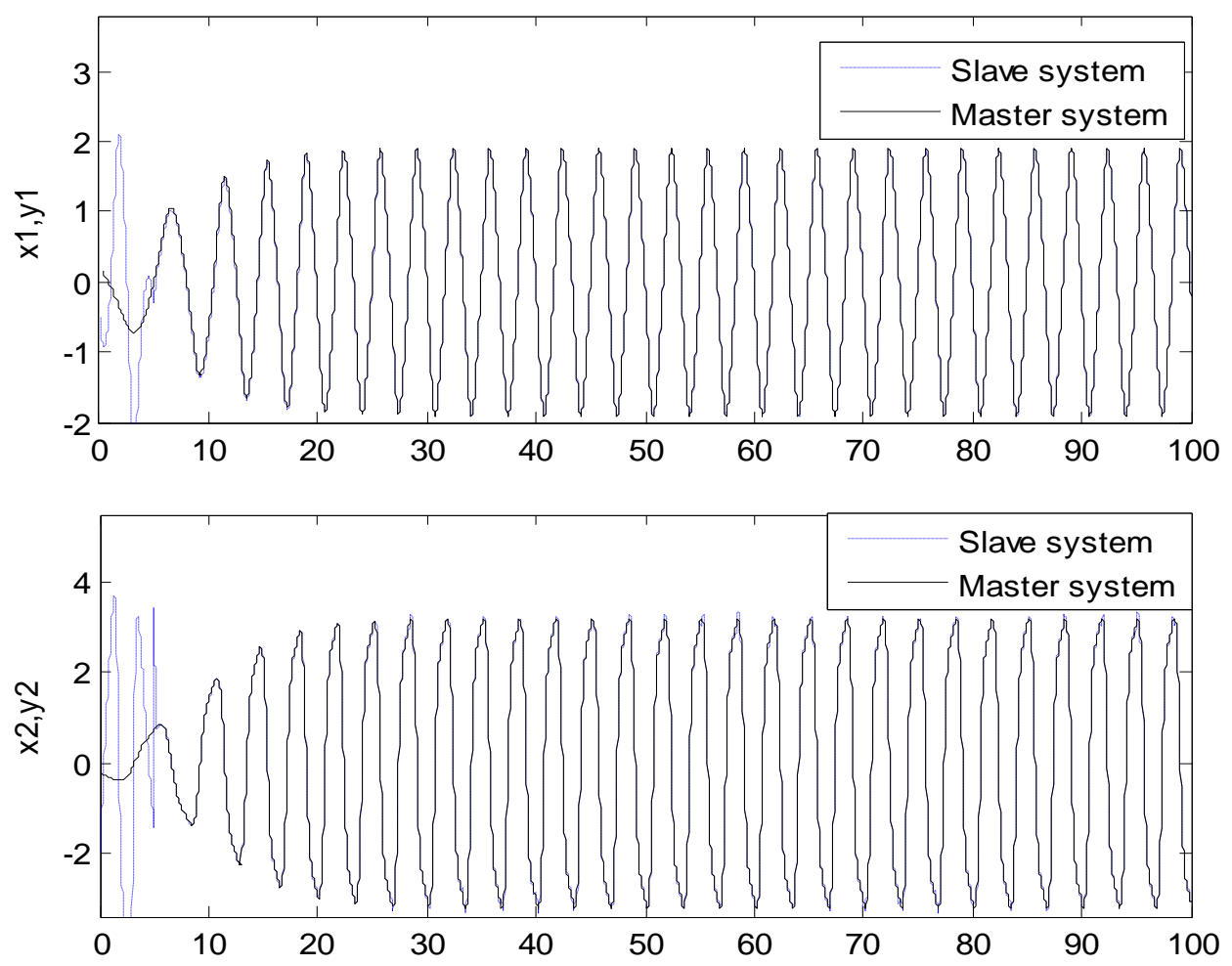

Figure 7: Time response of GBFC synchronization $\Phi 6-$ van der pol

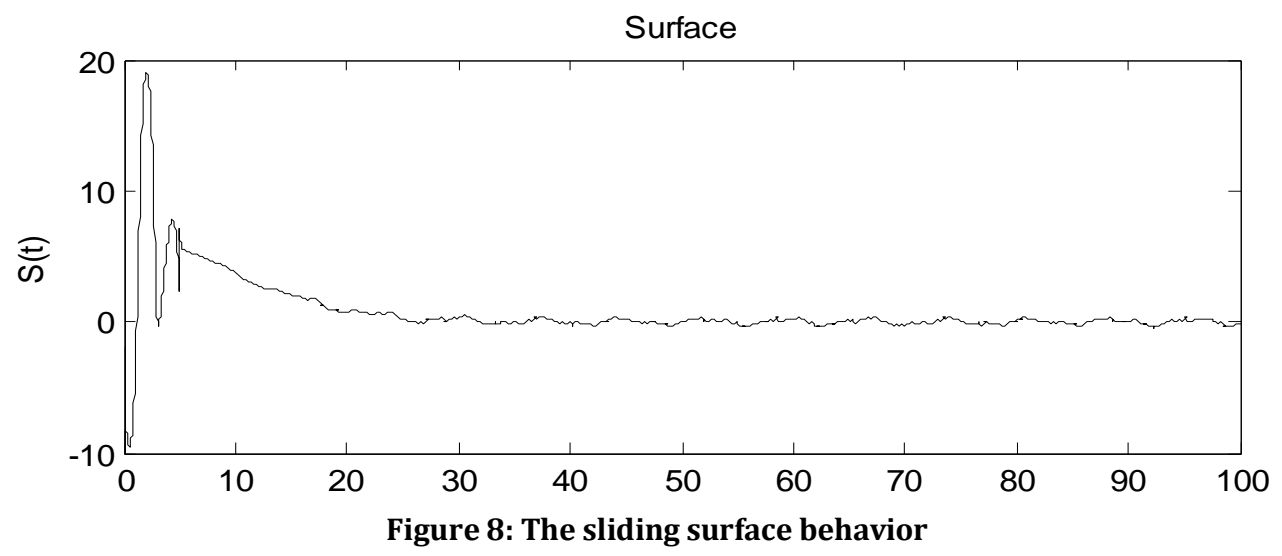




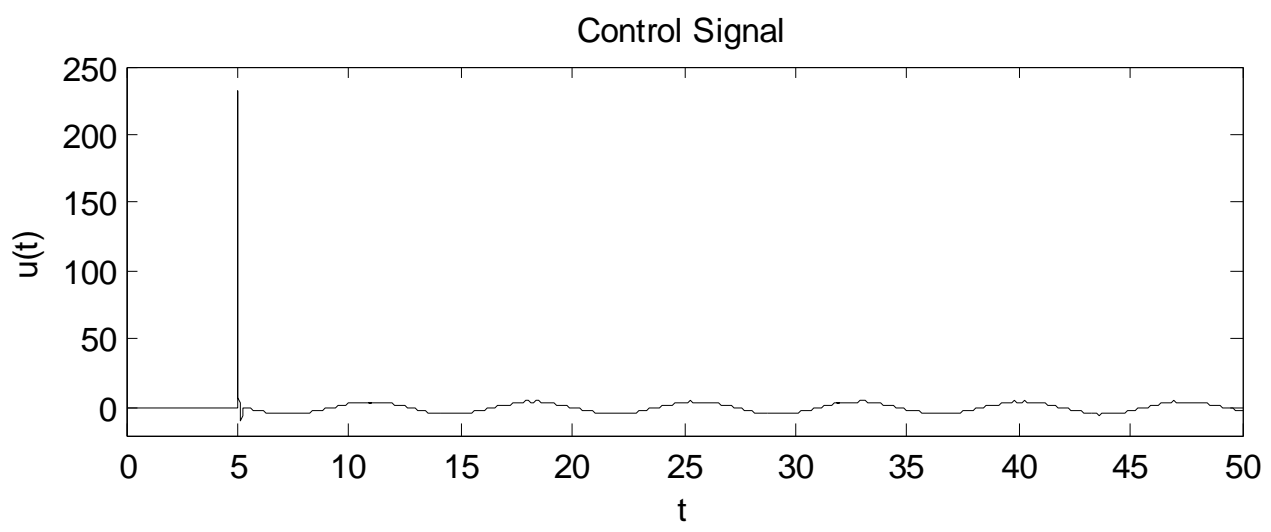

Figure 9: The control signal with GBFC
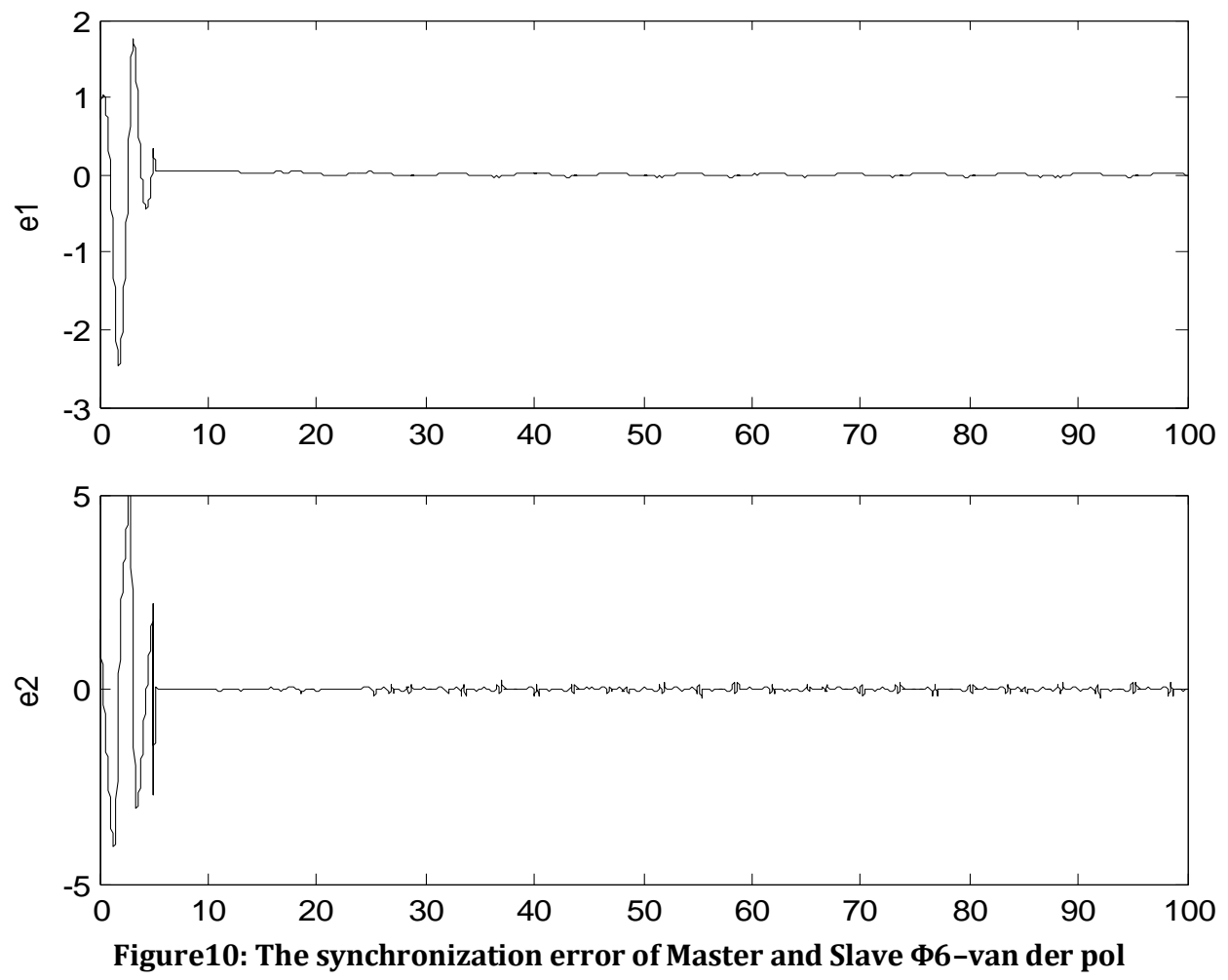

\section{Conclusion}

A methodology for synchronization of chaotic system in presence of uncertainty has been proposed. To deal the uncertainty, an Adaptive PID controller has been used on the slave system. A supervisory control has been used to guarantee the stability of the global system through energy like Lyapunov function. Furthermore, a sliding mode controller has been designed using another Lyapunov function to update the coefficients of the PID controller in an adaptive schema. The gradient method has been chosen to establish the adaptation law. The sign function in the supervisory controller cause the chattering phenomenon; to reduce this undesired effect a GBFC was proposed. The proposed synchronization method has been simulated on $\Phi^{6}$-Van Der Pol oscillator. The results confirm the significance of the proposed control technique. 


\section{References}

[1] Pecora, L.M. and T.L. Carroll, 1990. Synchronization in chaotic systems. Phys. Rev. Lett., 64: 821- 824.

[2] Yassen, M.T., 2005.The Chaos synchronization between two different chaotic systems using active control. Chaos Solitons Fractals, 23(1):131.

[3] Abdous, F. , A. Ranjbar N. , S. H. Hosein Nia, A. Sheikhol Eslami and B. Abdous, 2008. The Hopf Bifurcation control in Internet Congestion Control System. Second International Conference on Electrical Engineering (ICEE), Lahore (Pakistan) .

[4] Abdous, F., A. Ranjbar N., S. H. Hosein Nia and A. Sheikhol Eslami, 2008. Chaos Control of Voltage Fluctuations in DC Arc Furnaces Using Time Delay Feedback Control. Second International Conference on Electrical Engineering (ICEE), Lahore (Pakistan).

[5] Xie, Q. and G. Chen, 1996.Synchronization stability analysis of the chaotic Rossler system. Int. J. Bifur. Chaos 6(11):2153-2161.

[6] Kapitaniak, T., 1995.Continuous control and synchronization in chaotic systems. Chaos Solitons Fractals , 6(3): 237.

[7] Astakhov, VV., VS. Anishchenko, T. Kapitaniak and AV. Shabunin , 1997.Synchronization of chaotic oscillators by periodic parametric perturbations. Phys D 109: 11-6.

[8] Blazejczyk-Okolewska, B., J. Brindley, K. Czolczynski and T. Kapitaniak, 2001.Antiphase synchronization of chaos by noncontinuous coupling: two impacting oscillators. Chaos, Solitons \& Fractals 12:1823-6.

[9] Yang, XS., CK. Duan and XX. Liao,1999. A note on mathematical aspects of drive-response type synchronization. Chaos Solitons \& Fractals 10 : 1457-62.

[10] Wang, Y., ZH. Guan and X. Wen, 2004. Adaptive synchronization for Chen chaotic system with fully unknown parameters. Chaos, Solitons \& Fractals 19 : 899-903.

[11] Chua, LO., T. Yang, GQ. Zhong and CW. Wu, 1996. Adaptive synchronization of Chua's oscillators. Int J. Bifurc. Chaos 6(1): 189-201.

[12] Liao, TL., 1998. Adaptive synchronization of two Lorenz systems. Chaos, Solitons \& Fractals $9: 1555-61$.

[13] Lian, KY., P. Liu, TS. Chiang and CS. Chiu, 2002. Adaptive synchronization design for chaotic systems via a scalar driving signal. IEEE Trans Circuits Syst I 49(1):17-27.

[14] Wu, CW., T. Yang and LO. Chua, 1996. On adaptive synchronization and control of nonlinear dynamical systems. Int J Bifurc Chaos 6: 455-71.

[15] Fang JQ., Y. Hong and G. Chen,1999.Switching manifold approach to chaos synchronization. Phys Rev E 59: 2523-6.

[16] Yin, X., Y. Ren and X. Shan, 2002.Synchronization of discrete spatiotemporal chaos by using variable structure control. Chaos, Solitons \&Fractals 14 :1077-82.

[17] Yu, X. and Y. Song, 2001. Chaos synchronization via controlling partial state of chaotic systems. Int J Bifurc Chaos 11(6):1737-41.

[18] Wang, C. and SS. Ge, 2001. Adaptive synchronization of uncertain chaotic systems via backstepping design. Chaos, Solitons \& Fractals 12: 199-206.

[19] Lu“, J. and S. Zhang, 2001. Controlling Chen's chaotic attractor using backstepping design based on parameters identification. Phys Lett A 286: 145-9. 
[20] Suykens, JAK., PF. Curran, J. Vandewalle, 1997. Robust nonlinear synchronization of chaotic Lurenze systems. IEEE Trans Circuits Syst I 44(10) : 891-904.

[21] Wei-Der, Ch. and Y. Jun-Juh , 2005.Adaptive robust PID controller design based on a sliding mode for uncertain chaotic systems. Chaos, Solitons \& Fractals, 26(1): 167-175. [22] Yau, H-T and Ch. Chieh-Li, 2006.Chattering-free fuzzy sliding-mode control strategy for uncertain chaotic systems. Chaos, Solitons and Fractals 30: 709-718.

[23] Zhang, H., X-K. Ma and W-Z. Liu, 2004.Synchronization of chaotic systems with parametric uncertainty using active sliding mode control. Chaos, Solitons and Fractals 21: $1249-1257$.

[24] Tavazoei, M. S. and M. Haeri, 2007.Determination of active sliding mode controller parameters in synchronizing different chaotic systems. Chaos, Solitons and Fractals 32: 583-591.

[25] Yau, H-T., 2004. Design of adaptive sliding mode controller for chaos synchronization with uncertainties. Chaos, Solitons and Fractals, 22:341-347.

[26] Liang, C-Y. and J-P. Su, 2003. A new approach to the design of a fuzzy sliding mode controller. Fuzzy Sets and Systems 139: 111-124.

[27] Hung, JY. ,1995. Magnetic bearing control using fuzzy logic. IEEE Trans Indust Appl 31:1492-14927.

[28] Li, THS. and MY. Shieh, 2000. Switching-type fuzzy sliding mode control of a cart-pole system. Mechatronics 10: 91-109.

[29] Li, JH., THS. Li and TH. Ou, 2003. Design and implementation of fuzzy sliding-mode controller for a wedge balancing system. J. Intell. Robotic Syst., 37(3):285-306.

[30] Delavari, H. and A. Ranjbar, 2007. Robust Intelligent Control of Coupled Tanks. WSEAS International Conferences, Istanbul, Turkey, May 1-6.

[31] Delavari, H. and A. Ranjbar, 2007. Genetic-based Fuzzy Sliding Mode Control of an Interconnected Twin-Tanks. IEEE Region 8 EUROCON 2007 conference, Poland, September, 714-719.

[32] Chen, H.K., 2002. Chaos and chaos synchronization of a symmetric gyro with linearplus-cubic damping. Journal of Sound and Vibration, 255: 719-740.

[33] Yau, H-T., 2008. Chaos synchronization of two uncertain chaotic nonlinear gyros using fuzzy sliding mode control. Mechanical Systems and Signal Processing, 22(2): 408-418.

[34] F. M. Moukam Kakmeni and S. Bowong, C. Tchawoua and E. Kaptouom, 2004.Chaos control and synchronization of a $\Phi 6$-Van der Pol oscillator. Physics Letters A, 322 (5-6):305-323. 